\title{
EFFECT OF GANGLION IMPARE BLOCK ON CANCER PAIN CAUSED BY PUBIC BONE METASTASIS: REPORT OF 4CASE
}

T. Kosugi, M. Kubo, A. Ishikawa, T. Yumiba Department of Palliative Care, Saga-ken Medical Centre Koseikan, Japan

\section{Background and Aims}

In general, the indication of the ganglion impar block is considered to be the anal pain and the perineal pain.

We experienced accidentally that the ganglion impar block performed for the anal pain was effective for the pain caused by pubic bone metastasis. Furthermore, we went for this block for the pain caused by pubic bone metastasis, they were effective.
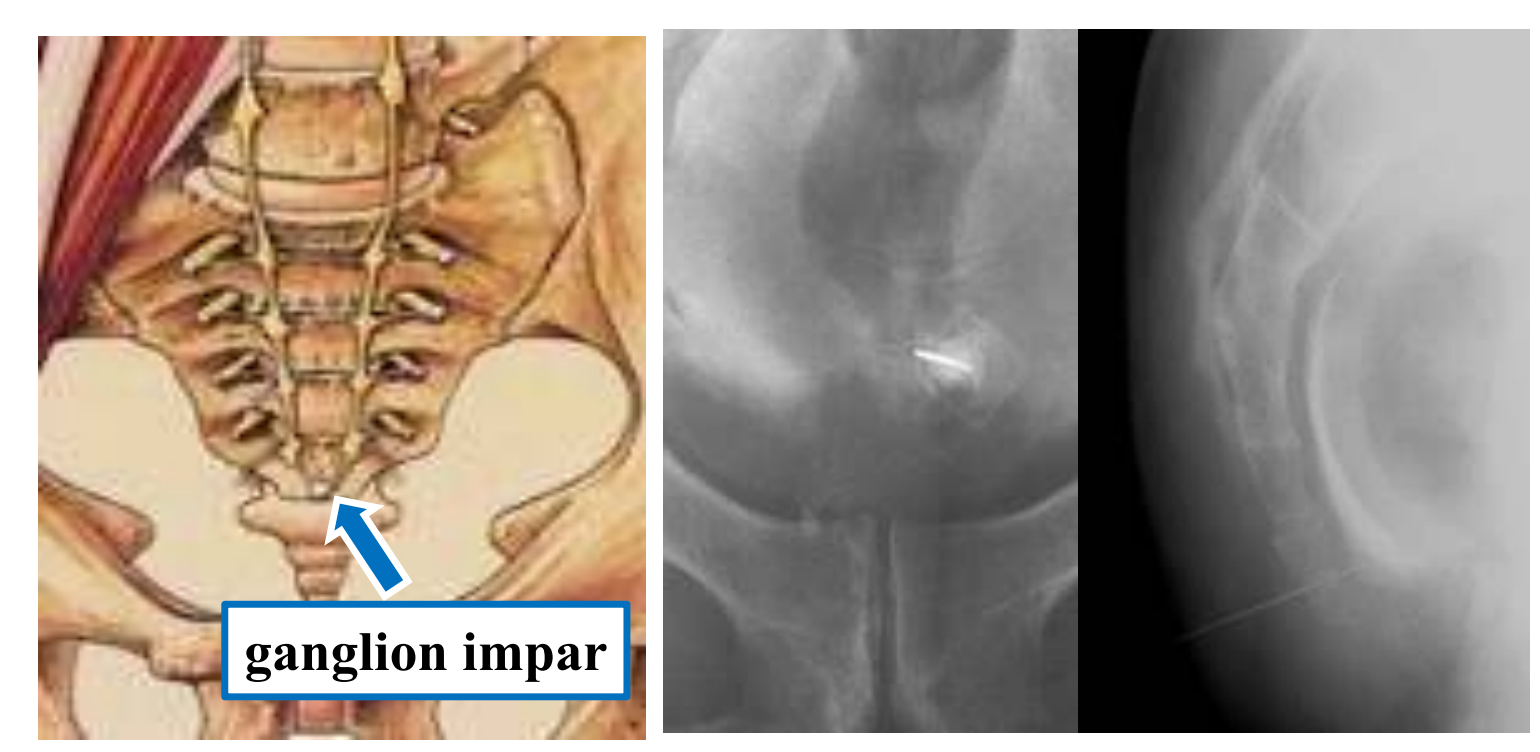

ganglion impar block

\section{Methods}

The ganglion impar block were performed for 4 cancer patients, all with cancer pain caused by pubic bone metastasis. The ganglion impar block with $3 \mathrm{ml}$ of $1 \%$ mepivacaine were performed at first. After successful good effects with local analgesic were obtain, neurolytic block were performed, by two times of radiofrequency thermocoagulation (at 90 degrees Celsius for 180 seconds) and injection of $3 \mathrm{ml}$ of absolute alcohol.

\section{Case1 58y.o. Female with Rectal cancer} \#1 Anal Pain caused by local invasion \#2 Inguinal pain caused by pubic bone metastasis At first, we perfomed the ganglion impar block for \#1. A very good analgesic effect for \#1 and \#2. (pre NRS9/10 $\rightarrow$ post NRS3/10)
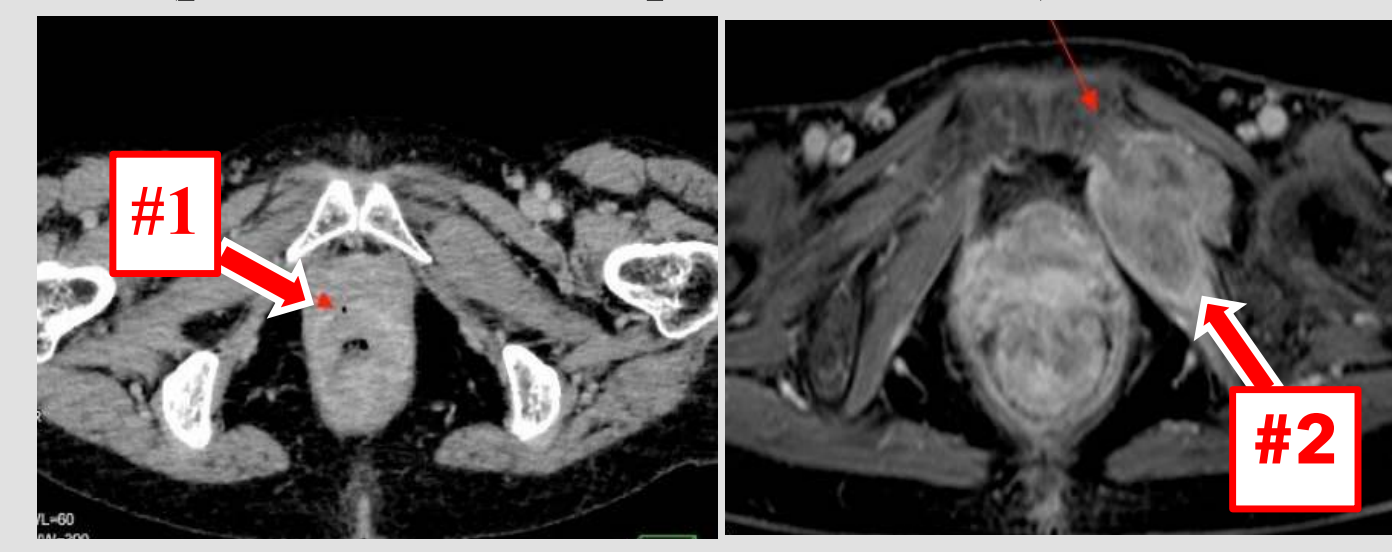

\section{Case367y.o. Female with Cholangiocarcinoma}

\#1 Inguinal pain due to pubic bone metastasis (pre NRS10/10 $\rightarrow$ post NRS0/10)
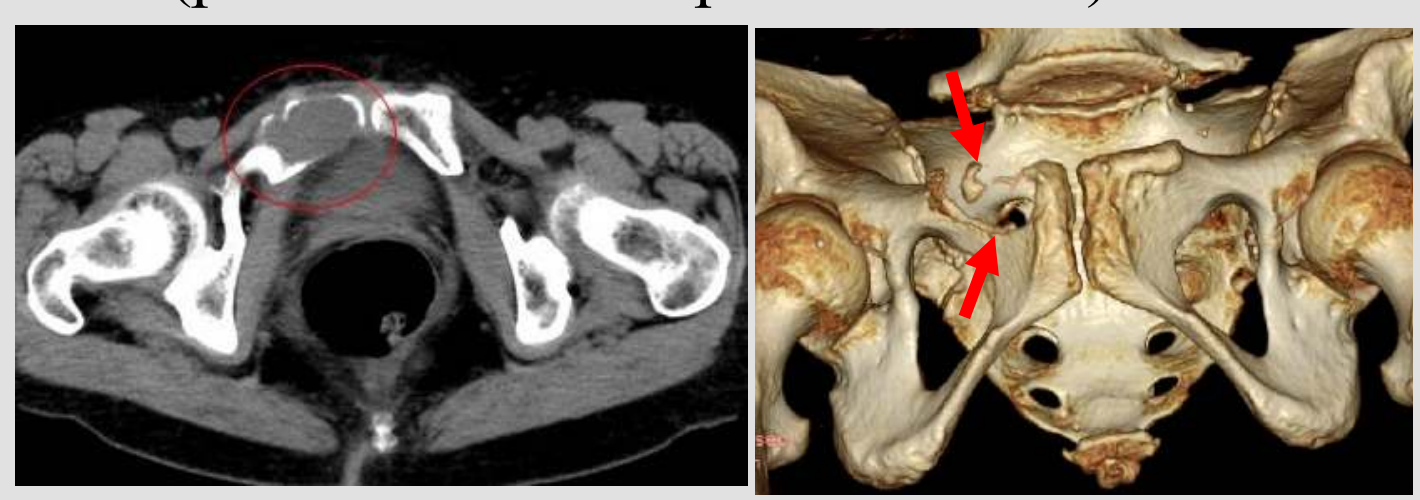

Case 2 72y.o. Female with endometrial cancer \#1 Inguinal pain due to pubic bone metastasis (decreased frquency of rescue doses of opioids: $5 /$ day $\rightarrow 1 /$ day)
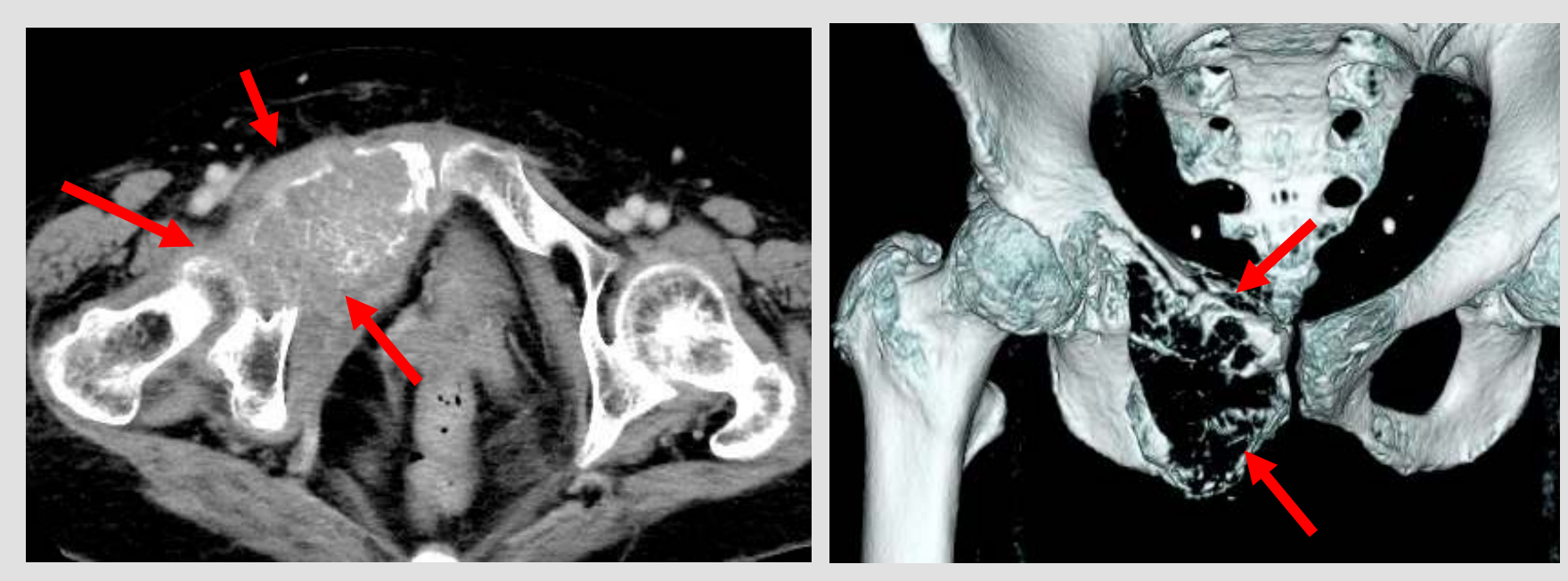

Case 4 70y.o. Male with Prostate cancer

\#1 Inguinal pain due to pubic bone metastasis (pre NRS9/10 $\rightarrow$ post NRS2/10)

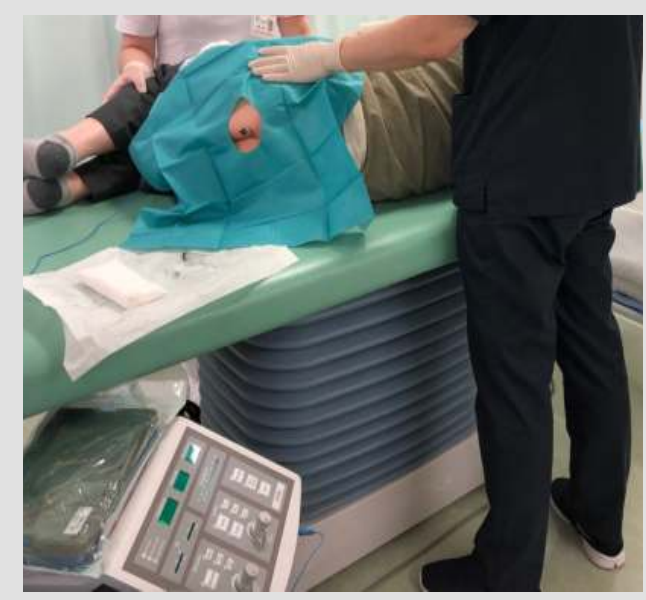

\section{Results}

All were female cancer patient from 58 years old to 72 years old. In three patients, NRS pain score were decreased, from mean NRS of 9.3(9/10-10/10) to mean NRS of $1.6(0 / 10-3 / 10)$. One patient obtained analgesic effect which was reduced the frequency for rescue dosage, from five times per day to one time per day.

\section{Disscussion}

Ganglion impar block is the most distal sympathetic nerve block. Why does this nerve block have a analgesic effect for not only anal pain but also pubic bone metastasis? The ganglion impar which is the most distal sympathetic nerve may participate in analgesic effect above it by some kind of mechanism.

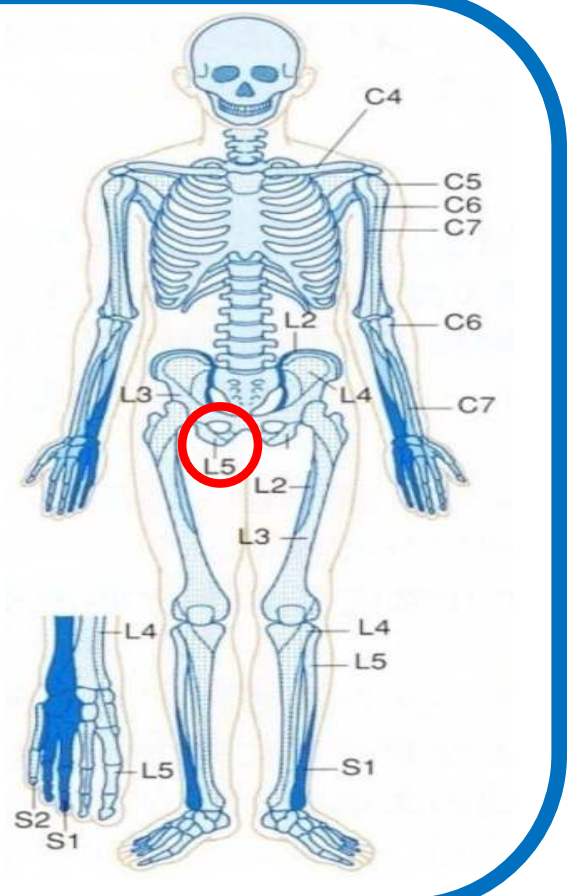

